\title{
MICROCHANNEL SPATIAL LIGHT MODULATOR
}

\author{
Cardinal Warde \\ Department of Electrical Engineering \\ and Computer Science \\ Massachusetts Institute of Technology \\ Cambridge, MA
}

SUMMARY

The Microchannel Spatial Light Modulator (MSLM) is a versatile, highly-sensitive, and optically addressed device that is being developed for real-time optical information processing. The MSLM operates by converting an input optical image into a charge distribution at the surface of an electro-optic crystal. The charge distribution generates an electric field which modulates the refractive index of the crystal and thereby the phase or intensity of an image readout beam. Prototype devices employing $250 \mu \mathrm{m}$ thick crystals have exhibited a spatial resolution of 5 cycles $/ \mathrm{mm}$ at $50 \%$ contrast, an exposure sensitivity of $2.2 \mathrm{~nJ} / \mathrm{cm}^{2}$ and framing rates of $40 \mathrm{~Hz}$ with full modulation depth. The image processing operations that have been achieved using the internal processing mode of the MSLM include contrast reversal, contrast enhancement, edge enhancement, image addition and subtraction, analog and digital intensity thresholding, and binary-level logic operations such as AND, OR, EXCLUSIVE OR, and NOR.

\section{INTRODUCTION}

The Microchannel Spatial Light Modulator (MSLM) is a relatively new, versatile, highly sensitive, and optically addressed device that is being developed for lowlight-level real-time optical information processing. In addition to real-time operation, it can be designed to exhibit long term (months) optical information storage simultaneously.

A variety of sophisticated image processing operations can be achieved using the internal processing mode of the MSLM. These include contrast reversal, contrast enhancement, edge enhancement, image addition and subtraction, analog and digital intensity level thresholding, as well as binary-level logic operations such as AND, OR, EXCLUSIVE OR, and NOR. This paper presents an update of its characteristics.

The MSLM is illustrated in Fig. 1. It consists of a photocathode, a microchannel. plate (MCP), a planar acceleration grid (optional), and an electro-optic plate in a proximity focussed configuration. The electro-optic plate generally carries a high-resistivity dielectric mirror on one side and a transparent conducting electrode on the other. The MCP is an array of small $(\sim 10 \mu \mathrm{m})$ semiconducting-glass pores, each functioning as a continuous dynode electron multiplier2,3. Spatially uniform gains ( $+5 \%$ variation) of $10^{4}$ with a single MCP or $10^{7}$ with two cascaded MCPs (chevron configuration) are typical? 
In operation, the write light (coherent or incoherent) incident on the photocathode creates an electron image which is amplified by the MCP and proximity focused onto the surface of the electro-optic plate. The resulting surface charge distribution creates a spatially varying electric field which modulates the refractive index of the electro-optic plate (crystal). Thus the readout light, which makes a double pass through the crystal, is spatially phase modulated.

It is the wide choice of photocathodes and electro-optic materials that gives rise to the spectral versatility of the MSLM and permits its characteristics to be tailored for specific applications. For example, the wavelength of the write signal is limited only by the spectral response of existing photocathodes. No photocathode is needed in the extreme Ultraviolet and $X$-ray regions since the MCP responds direct$1 y$ to this radiation 5,6 . The readout light is limited by the spectral transmittance of electro-optic materials (the range between the middle ultraviolet and the middle infrared).

Because of its large electron gain and ability to integrate charge, the MSLM is capable of achieving the high photon sensitivities of image intensifier tubes. Devices employing one MCP are expected to be able to record visible images with intensities as low as $10^{-14} \mathrm{~W} / \mathrm{cm}^{2}$, the primary limitation being MCP dark current.

\section{PRINCIPLES OF OPERATION}

The dynamics of the write and erase processes in the MSLM can be understood from a study of the electron current in the gap between the grid and the crystal. The gap current density $\mathrm{Jg}$ depends on the voltage $V_{g}$ at the crystal surface, the secondary emission characteristics of the crystal surface and on the MCP output electron energy distribution.

Figure 2 summarizes the dependence of the gap current on the above-mentioned parameters. The parameter $\bar{\delta}^{\prime}\left(V_{k}\right)$ is an effective secondary electron emission coefficient (for electrons removed from the crystal) that takes the MCP energy distribution and the collection geometry into account. The point $E$ in Figure 2 is an equilibrium point, and $M$ is the point at which electrons can be removed from the crystal at the fastest rate. If the device is driven into lockout regime by lowering $V_{b}$ too rapidly, the crystal surface repels the primary electrons from the MCP and it becomes very difficult to alter the state of the device.

The MSLM is therefore operated so that it is either at the equilibrium point E in Fig. 2, or it is moving along one of the trajectories of Fig. 2 toward equilibrium. For materials exhibiting the linear electro-optic effect, two write modes are possible. In the electron deposition write mode, $V_{b}$ initially divides capacitively between the crystal and the gap, thereby raising $V_{g}$ into the region for which $\bar{\delta}^{\prime}<1$. Then when the write light is turned on, electrons are deposited on the crystal, $V_{g}$ begins to fall toward $i$ ts equilibrium value $V_{e g}$, and the crystal voltage $V_{x}$ rises. Electron accumulation may be terminated when the desired $V_{X}$ is reached by turning off the write light. The crystal may be erased via secondary electron emission by flooding the photocathode with light and lowering $V_{g}$ (by lowering $V_{b}$ ) into the region where $\bar{\delta}^{\prime}>1$. In practice, $V_{b}$ is ramped downward no faster than $V_{g}$ can be restored to $V_{e q}$ by 
electron removal, so as to avoid lockout.

In the secondary electron emission write mode, with the write light on, $V_{b}$ is ramped downward at a rate $\dot{V}_{\mathrm{b}}$ chosen so that lockout is avoided and the appropriate electron distribution is removed from the crystal. The secondary emission write process terminates when either the write light is turned off, or $V_{b}$ reaches its final value and $V_{g}$ returns to its equilibrium value. To erase the information, $V_{b}$ can be increased abruptly and the photocathode flooded with light so that the crystal gains electrons.

Optical information that has been written into the MSLM may be read out either interferometrically with schlieren optics or with crossed polarizers. The possible readout modes depend on the symmetry and cut of the electro-optic crystal. The readout mode that is used in the prototype devices employs the interference between the front and back surface reflections from a plane-parallel electro-optic crystal. If dielectric mirrors are deposited on both sides of such a plane-parallel crystal to form a Fabry-Perot interferometer of finesse F, a complete off-to-on transition can be achieved with approximately $1 / F$ times the halfwave surface charge density of the crystal.

The MSLM can be either framed through a discrete sequence of write, read, and erase periods or operated in a continuous mode. In the framed mode, the optica 7 write signal is integrated and stored in the form of a charge distribution; after the modulation is read out, the charge distribution is removed. In the continuous mode of operation, which can be achieved with low resistivity crystals, the instantaneous modulation is proportional to the write image intensity for all temporal variations within the bandwidth of the system.

\section{CHARACTERISTICS OF PROTOTYPE DEVICES}

The most recent vacuum-demountable prototype devices built at M.I.T. employed $0.25 \mathrm{~mm}$ - thick oblique-cut $\mathrm{LiNbO}_{3}$ crystals coated on one side with a dielectric mirror and on the other with a transparent indium-tin-oxide electrode. A 25-mm diameter MCP was used in these devices, and the metal electrode on the input face of the MCP served as the photocathode for the $254 \mathrm{~nm}$ write 1 ight.

The spatial resolution of these devices was approximately 5 cycles $/ \mathrm{mm}$ at $50 \%$ contrast, and image degradation after several hours of storage time was negligible. Figure 3 shows how the spatial resolution of the MSLM depends on device geometry, and Fig. 4 shows a sample of some of the image processing operations that have been achieved with a vacuum demountable device. The sequence of steps for achieving these operations are described in Ref. 1.

In an earlier vacuum demountable device employing a Z-cut LiNb0 3 Fabry Perot crystal, a framing rate of $40 \mathrm{~Hz}$ at full modulation depth was achieved. Additionally, we have observed an exposure sensitivity of $2.2 \mathrm{~nJ} / \mathrm{cm}^{2}$ at $655 \mathrm{~nm}$ wavelength in a vacuum sealed device employing a bialkali photocathode and a Z-cut $\mathrm{LiTaO}_{3} \mathrm{Crystal}$ $0.5 \mathrm{~mm}$ thick. This device exhibited extremely long image storage times as shown by the history of image degradation shown in Fig. 5. 


\section{COMMENTS}

The resolution characteristics of the MSLM have been improved by reducing the thickness of the crystal. Further improvements in resolution are expected in the near future. The large class of image processing operations that can be achieved by internal processing and the demonstrated potential for kilohertz framing rates are expected to make the MSLM useful for a large number of signal processing operations.

\section{REFERENCES}

1. C. Warde, A. M. Weiss, A. D. Fisher and J. I. Thackara, "Optical Information Processing Characteristics of the Microchannel Spatial Light Modulator," Applied Optics 20, 2066-2074, 1981.

2. B. Leskovar, Phys. Today 30, 42, No. 11, Nov. (1977).

3. J. L. Wisa, Nuc7. Instrum. Methods 162, 587 (1979).

4. Applications for Microchannel Plates, Varian Associates, Palo Alto, Calif.

5. P. J. Bjorkholm, L. P. VanSpeybroeck, and M. Hecht, Proc. Soc. Photo-Opt. Instrum. Eng. 108, 189 (1977).

6. K. W. Dolan and J. Chang, Proc. Soc. Photo-0pt. Instrum. Eng. 106, 178 (1977). 


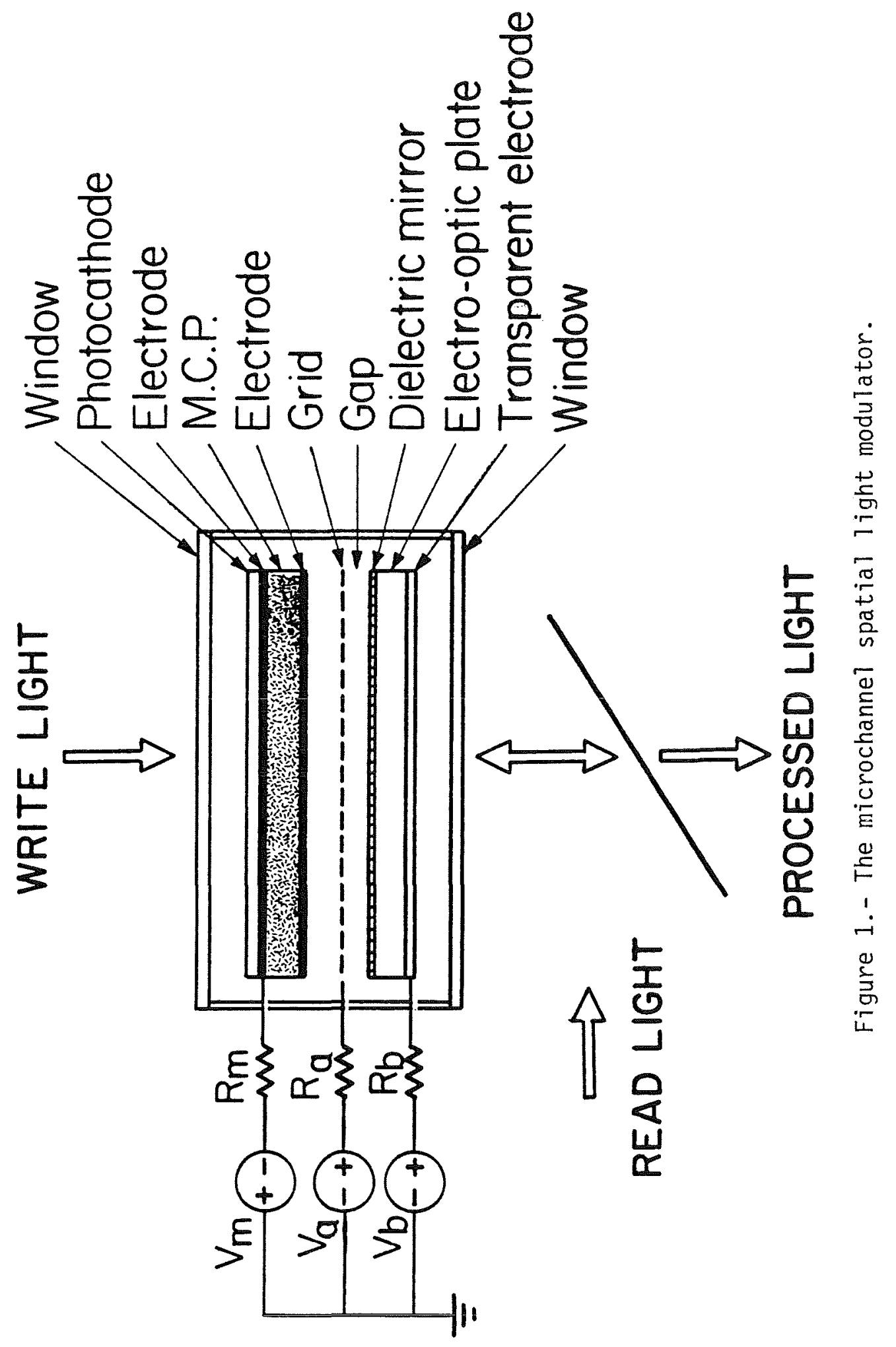




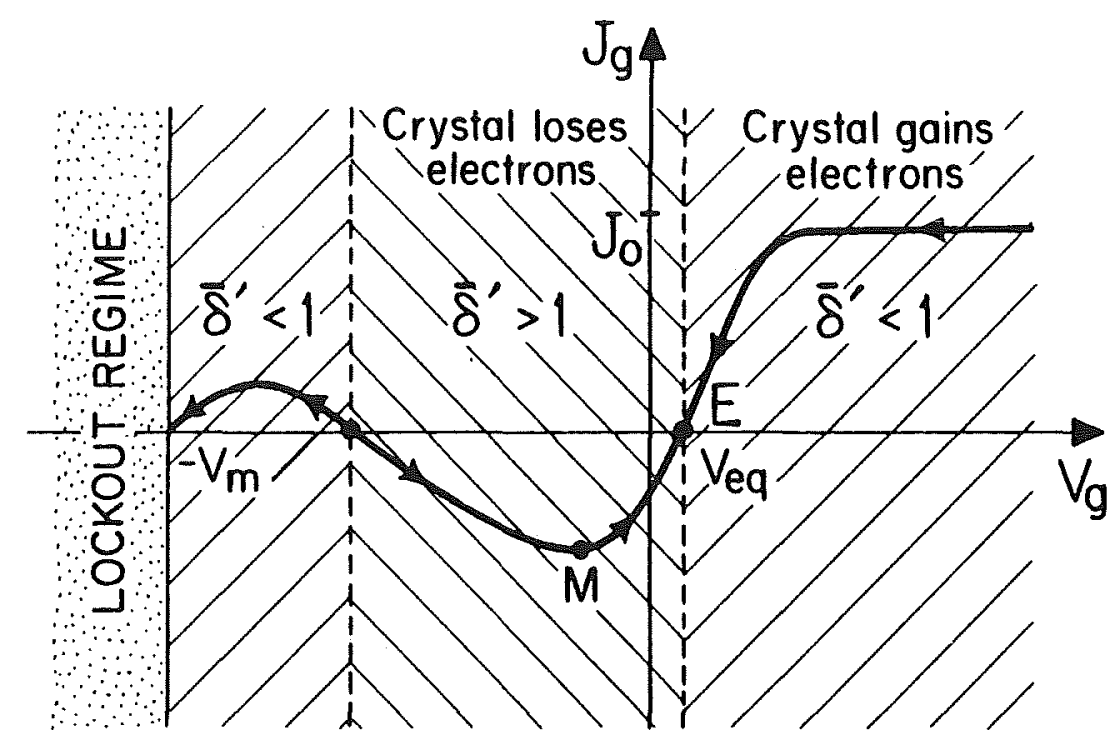

Figure 2.- Sketch of gap current density $\mathrm{J}_{\mathrm{g}}$ in an MSLM as a function of gap voltage $V_{g}$ for constant write light intensity $I$ and MCP bias voltage $-V_{m}$.

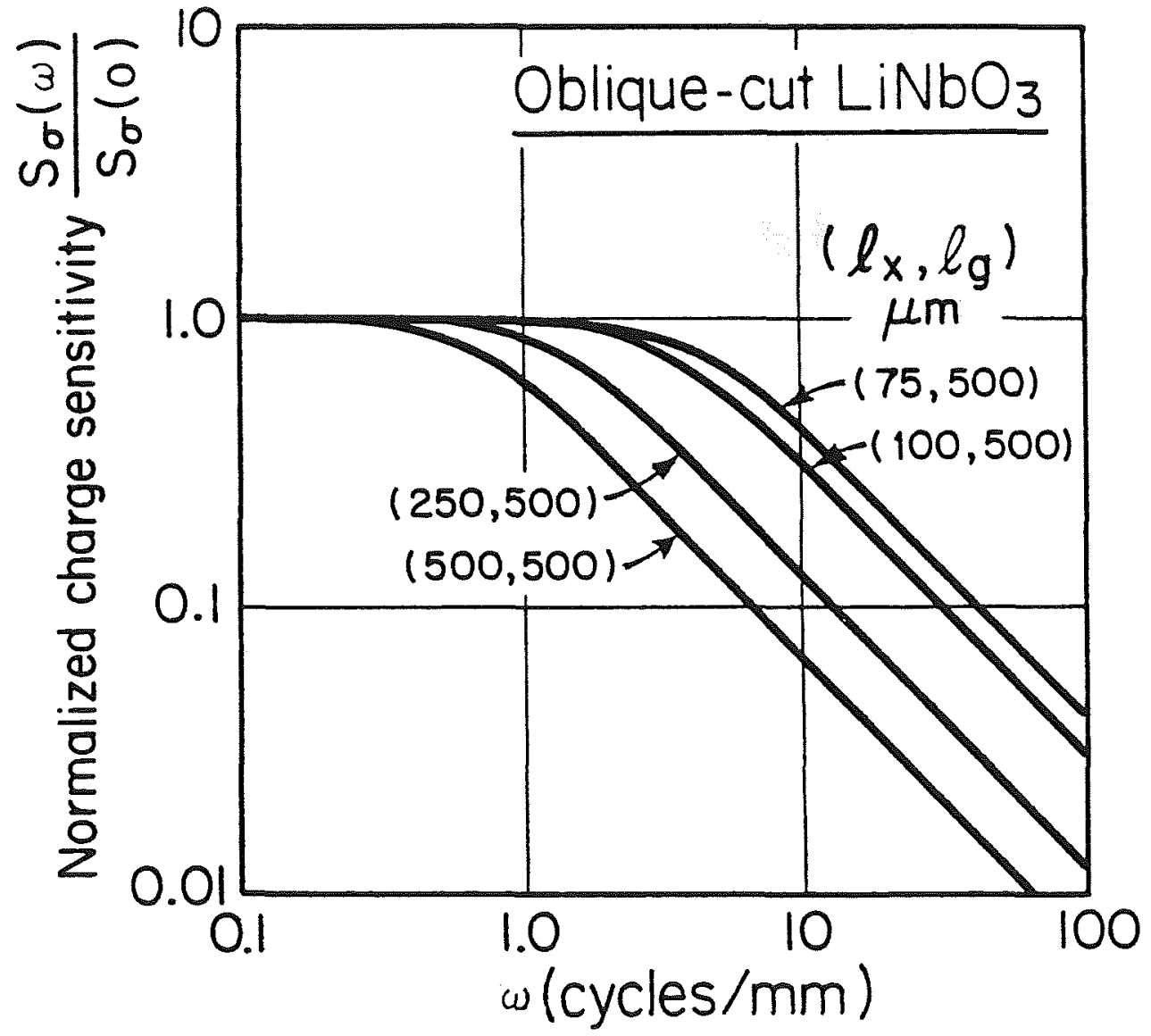

Figure 3. - Calculated normalized charge sensitivity $S_{\sigma}(\omega) / S_{\sigma}(0)$ plotted against spatial frequency $\omega$ for an oblique-cut $\mathrm{LiNbO}_{3}$ MSLM with crystal thickness and gap width as parameters. 

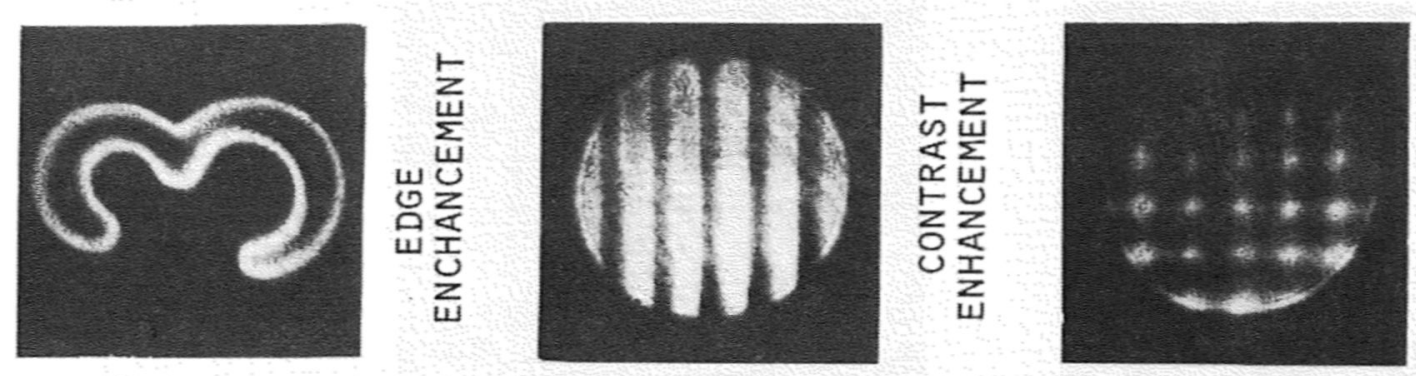

$\frac{0}{2}$
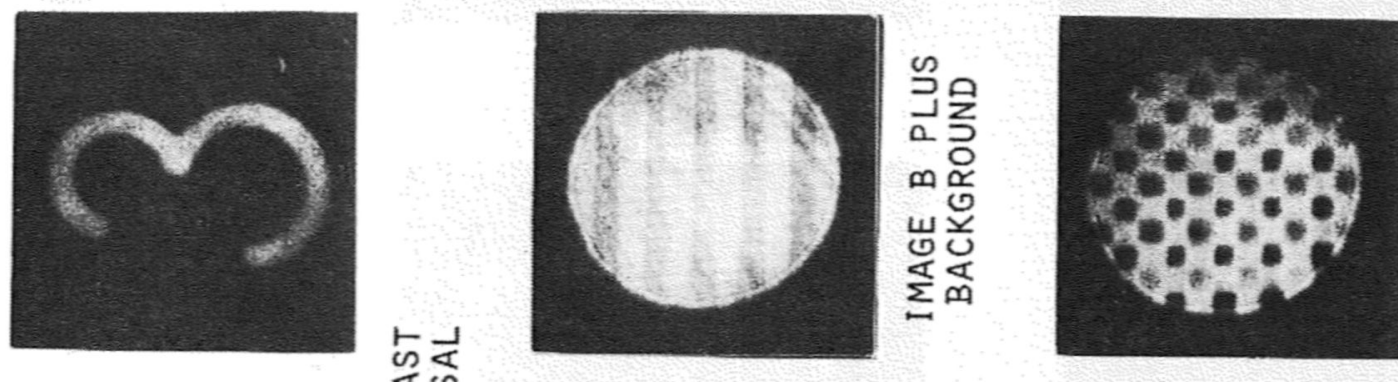

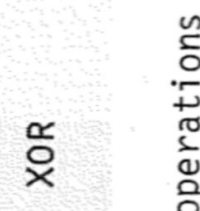

षक

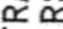

它岁
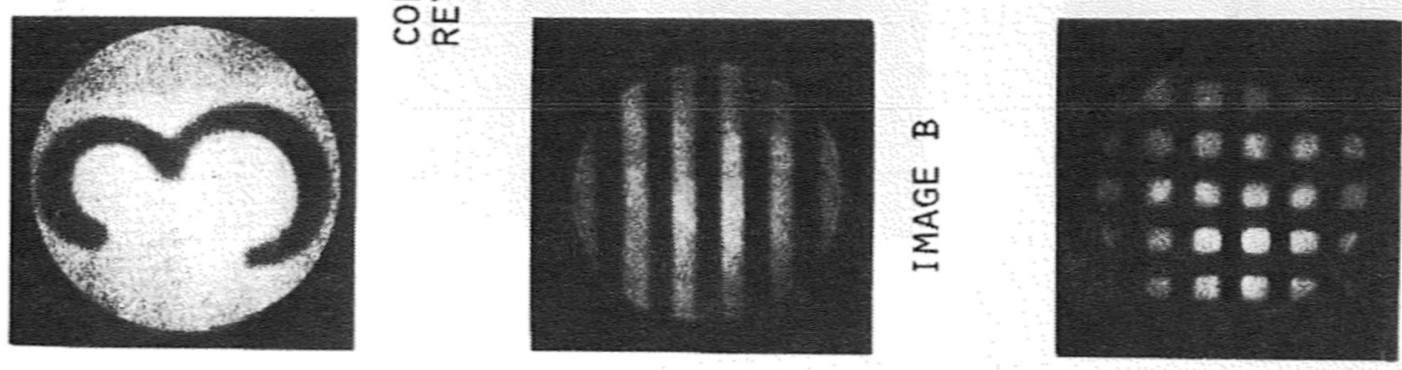

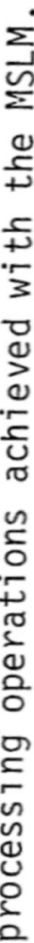

따
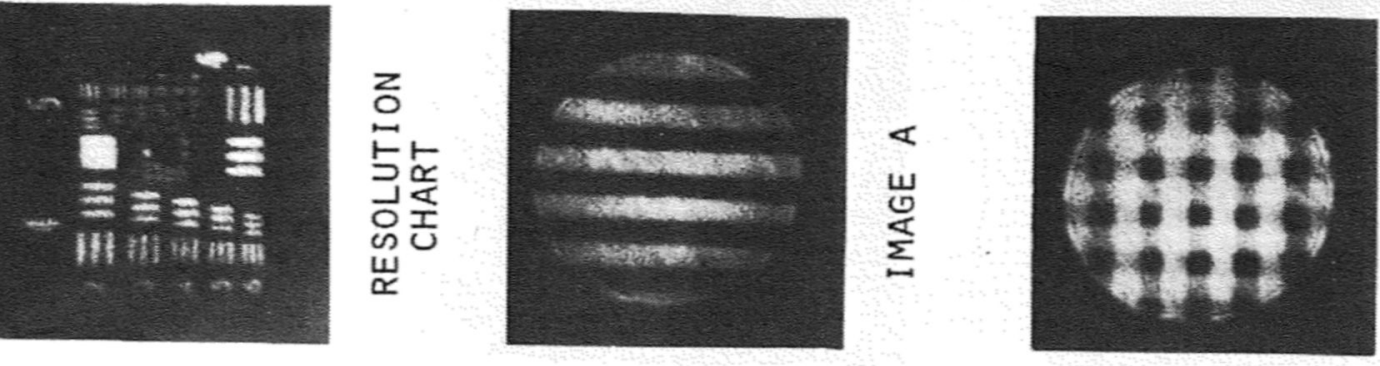

岕兑 

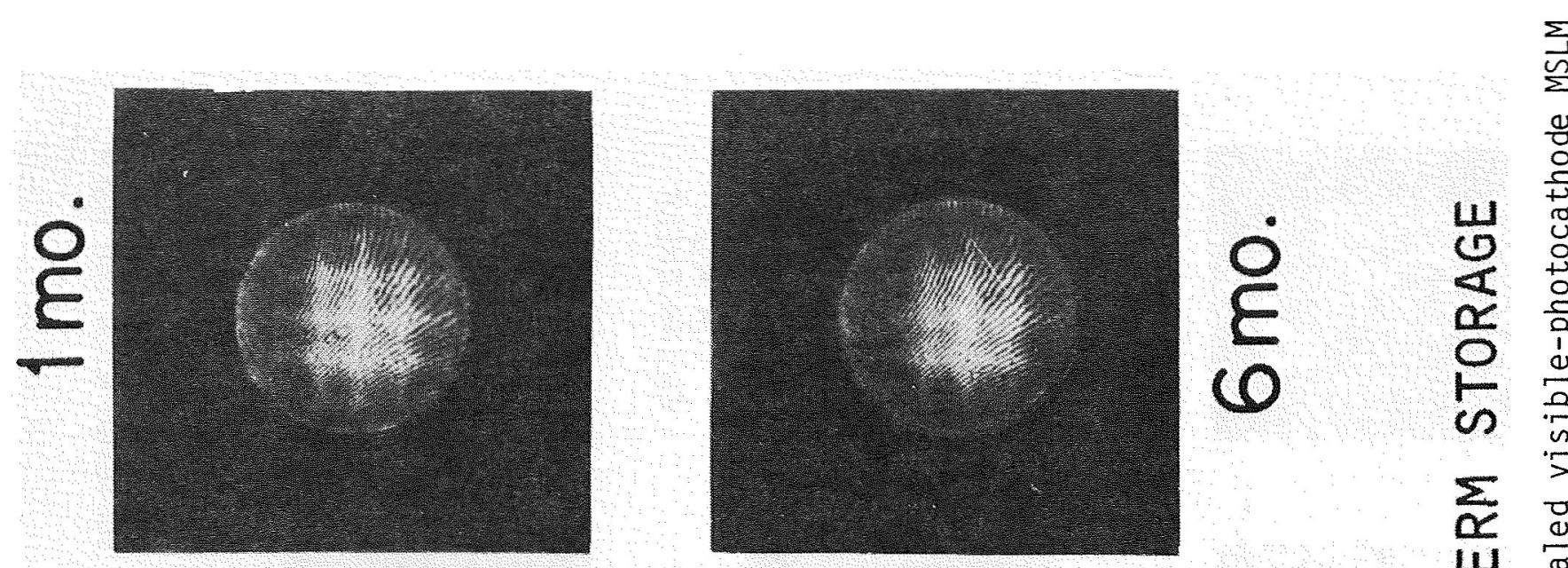

$\frac{\sum}{n}$

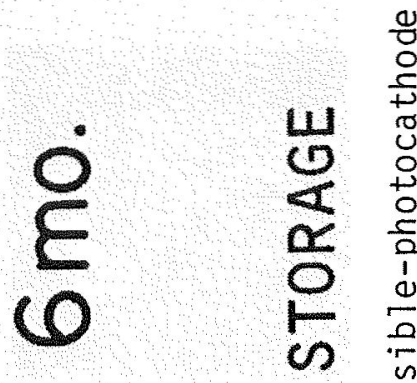

$\infty$ is

$\sum>$

u

$1 \quad 0$
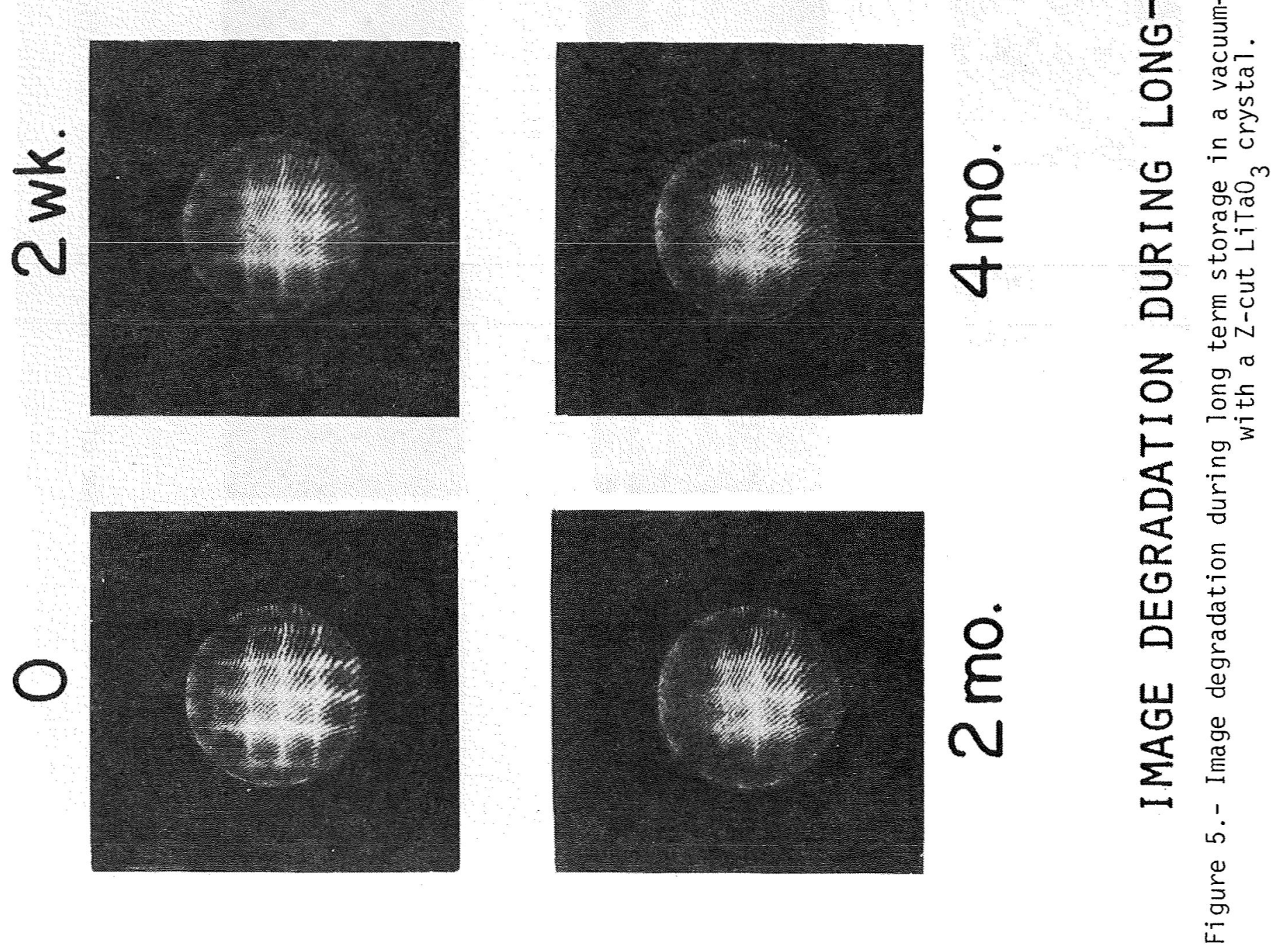